\title{
UV written evanescent devices fabricated in micro-structured substrates for optofluidics.
}

\author{
James C. Gates, Christopher Holmes, Benjamin D. Snow, Corin B.E. Gawith and Peter G.R. Smith \\ Optoelectronics Research Centre, University of Southampton, UK \\ jcg@orc.soton.ac.uk
}

\begin{abstract}
We report our recent developments in micro-structured evanescent devices. Micromachining prior to direct UV writing of channel waveguides provides additional flexibility to fabricate evanescent interacting devices such as modulators and sensors, which allow adiabatic operation.

(C) 2008 Optical Society of America

OCIS codes: (280.4788) Optical sensing and sensors; (130.3120) Integrated optics devices.
\end{abstract}

Evanescent channel waveguide devices provide a route to realizing refractive index sensors and modulators. In the past gaining access to the evanescent field of the waveguide has been achieved via etching of the cladding layer. In this work an alternative geometry presented, where a vertical trench or groove is pre-defined to provide an interface of optical quality for lateral access for an optical mode. A channel waveguide may then be UV written in proximity to the groove to allow the evanescent field of an optical mode to interact with the material within the groove. Interrogation of the local refractive index is provided by a Bragg grating simultaneously defined within the region during the UV writing process. This relatively subtle change to substrate engineering not only simplifies the fabrication process, by removing the need for the commercially undesirable process of etching, but more importantly provides an extra degree freedom to tailor the interaction between the optical mode and the surrounding material. This interaction may differ in penetration depth, sensitivity, transverse mode and Bragg grating design. The process also provides other potential benefits including a reduction in return loss, spectral artifacts and stress induced birefringence. The groove also acts as a microfluidic channel for liquid delivery to realize optofluidic devices.

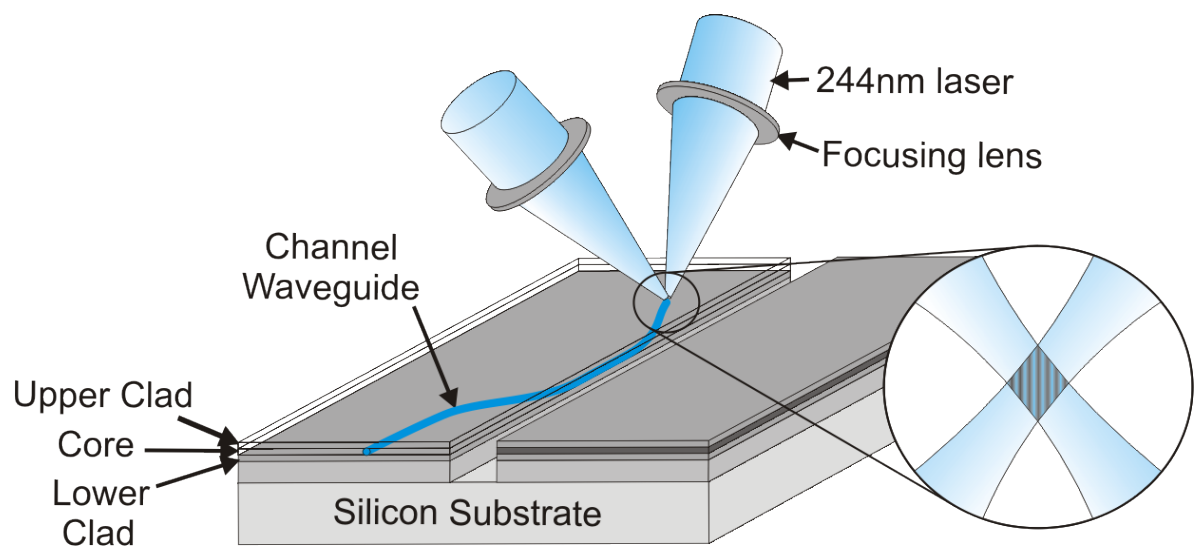

Figure 1: Illustration of the successful fabrication process using micro-structured substrates and UV writing. The gradual approach of the waveguide to the micro-structured groove allows adiabatic operation.

The fabrication process is made possible due to the unique method of fabricating waveguides and Bragg gratings called direct UV writing. A diagram of the process is shown in Figure 1, illustrating the dual beam method used to fabricate channel waveguides with integral Bragg gratings [1]. The planar samples consist of three layers of silica deposited on a silicon substrate with the core layer made photosensitive by germanium doping. In order to define a Bragg grating an interference pattern is generated by the crossing of two beams beneath which the sample is stepped to build up a periodic $\Delta \mathrm{n}$ pattern in the photosensitive glass. In this case the source of light is a frequency doubled Argon Ion laser (244nm). The core layer is exposed to this interference pattern while the sample is moved to generate the desired optical waveguides. Bragg grating properties such as position, period and contrast are controlled by the synchronized modulation of the writing laser. Figure 2(a) shows an example of this control through the reflection 
spectra of two gratings within a single waveguide. The shorter wavelength grating having uniform apodization, where as the longer wavelength has sine apodization.

Figure 1 also shows the basic concept of the groove geometry evanescent device. Here, a UV written channel waveguide is defined approaching into and out off proximity with the microfluidic groove via two S-bends. This lateral control of the waveguides position and size provides a greater control over the design of the devices. There are several additional advantages to arise from this technique. In an etched sensor the change from the normal waveguide to sensor waveguide happens very rapidly meaning that the resulting step in propagation constant produces undesirable back reflection and cavity effects. However, in our groove based devices the s-bend provides an essentially adiabatic change in propagation constant, reducing these unwanted effects.

Another novel enabling technology employed in this work is the use of micro-machining to produce the microfluidic grooves. The machining is performed using polishing/dicing technology developed for silicon wafer processing, which has had little prior application to the fabrication of planar waveguides [2]. Recent improvements to the process means that the surface quality of the channels are of sufficient optical quality. And example of a sample with a series of micro-machined grooves is shown in Figure 2(b).

(a)

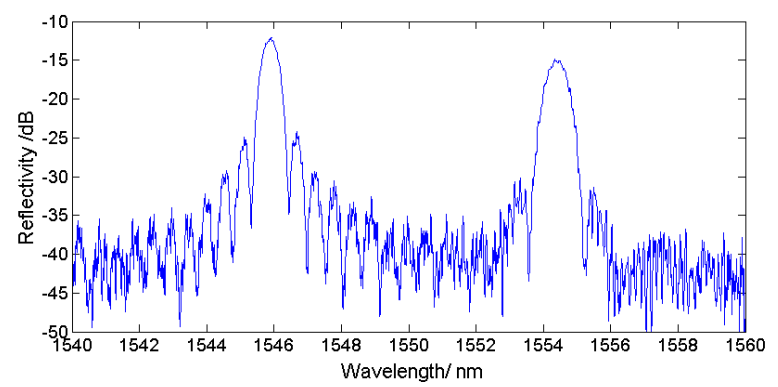

(b)

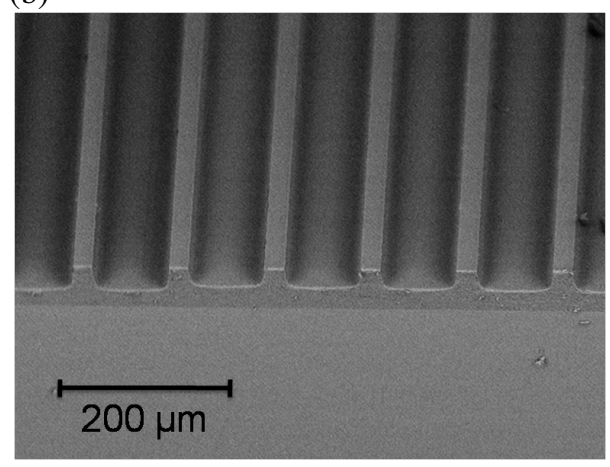

Figure 2: (a) Shows the reflectance of two Bragg gratings written into a single channel, and (b) is a scanning electron microscope image of a micro-structured substrate using the dicing/polishing technology.

The author will present novel device architectures and features of our latest evanescent groove devices including refractive index sensors in the telecom region $(1.55 \mu \mathrm{m})$. Our first devices have demonstrated detection of refractive index changes of $\approx 10^{-4}$ and $\approx 10^{-5}$ at indices of 1.33 and 1.45 respectively. Also reported on is the demonstrated of an electrically tunable modulator employing liquid crystal as the active liquid. Advantages of the technique in terms of device design, fabrication and optimization will also be discussed.

This work introduces an alternative method for fabricating planar evanescent waveguide devices. Current results will be discussed looking at the sensitivity of such devices for sensor applications and additional flexibility which provides a method to realize optofluidic devices previously difficult to fabricate. Also reported are investigations into the use of polishing/dicing saw technology developed for silicon wafer processing to produce channels with a vertical side wall finish sufficient for modal interaction. Using this technique refractive index sensors and liquid crystal modulators have both been demonstrated. This work has implications for use in the areas of distributed sensing and planar lightwave circuits.

\section{References}

[1] G. D. Emmerson, S. P. Watts, C. B. E. Gawith, V. Albanis, M. Ibsen, R. B. Williams, and P. G. R. Smith. Fabrication of directly UV-written channel waveguides with simultaneously defined integral Bragg gratings. Electronics Letters, 38(24):1531-1532, 2002.

[2] M. Iwai, T. Yoshino, S. Yamaguchi, M. Imaeda, N. Pavel, I. Shoji, and T. Taira. High-power blue generation from a periodically poled $\mathrm{MgO}: \mathrm{LiNbO}_{3}$ ridge-type waveguide by frequency doubling of a diode end-pumped $\mathrm{Nd}_{2} \mathrm{Y}_{3} \mathrm{Al}_{5} \mathrm{O}_{12}$ laser. Applied Physics Letters, 83(18):3659-3661, 2003. 